\title{
REFERENCES
}

1. Z. Nehari, On the singularities of Legendre expansions, J. Rat. Mech. Anal. 5 (1956), 987-992.

2. S. Bergman, Zur Theorie der algebraischen Potentialfunktionen des dreidimensionalen Raumes, Math. Ann. 99 (1928), 629-659.

3. R. Gilbert, Singularities of three-dimensional harmonic functions, Pacific J. Math. 10 (1960), 1243-1255.

4. J. Hadamard, Theorème sur les séries entières, Acta Math. 22 (1898), 55-64.

Michigan State University

\section{AN ALGORITHM OF J. SCHUR AND THE TAYLOR SERIES}

E. H. CONNELL AND P. PORCELLI

In a recent announcement in the Bulletin (cf. [1]), the authors outlined a proof that a differentiable function of a complex variable in a region $R$ has a power series expansion in some neighborhood of each point of $R$ (Theorem 3, [1]). The proof involved no integration, but failed to yield the full radius of convergence and required that it first be shown that all of the derivatives exist. In the last paragraph of [1], it was mentioned that a variation of an algorithm of J. Schur develops constructively the Taylor series for $f$, yields the full radius of convergence, and gives the existence of all the derivatives of $f$ as a corollary. This procedure is developed in this paper.

The letter $R$ will denote a bounded region in the complex plane.

Lemma 1 (cf. $[4$, p. 77$]$ ). If $f: \bar{R}$ into $E_{2}$ is continuous on $\bar{R}$, differentiable on $R$, and nonconstant, then

$$
|f(z)|<\max _{t \in \bar{R}-R}|f(t)| \quad \text { for all } z \in R .
$$

Lemma 2 (Theorem 1, [1]). If $p \in R$ and $f$ is differentiable and bounded on $R-p$, then $f$ may be defined at $p$ so that it will be continuous and differentiable at $p$.

LEMma 3. If the polynomial, $P(z)=a_{0}+a_{1} z+\cdots+a_{n} z^{n}$, satisfies $|P(z)| \leqq 1$ for $|z| \leqq 1$, then $\left|a_{i}\right| \leqq 1, i=0,1, \cdots, n$.

Proof. The conclusion holds for all polynomials of degree zero. Suppose it holds for all polynomials of degree $\leqq k$ and $P(z)=a_{0}$

Received by the editors March 1, 1961. 
$+a_{1} z+\cdots+a_{k+1} z^{k+1}$ with $|P(z)| \leqq 1$ for $|z| \leqq 1$. If $Q(z)$ $=2^{-1}\left[P(z)-P\left(e^{i \theta} z\right)\right], 0 \leqq \theta<2 \pi$, then $|Q(z)| \leqq 1$ for $|z| \leqq 1$. Now $Q(z)$ has no constant term and, consequently, $z^{-1} Q(z)$ is a polynomial of degree $\leqq k$. Note that $\left|z^{-1} Q(z)\right| \leqq 1$ for $|z|=1$ and by Lemma 1 is $\leqq 1$ for $|z| \leqq 1$. By the inductive hypothesis, the coefficients of $z^{-1} Q(z)$ are $\leqq 1$ in modulus. By letting $\theta=\pi n^{-1}$ we get $\left|a_{n}\right| \leqq 1$ for $n=1,2, \cdots, k+1$. Finally, $\left|a_{0}\right|=|P(0)| \leqq 1$.

LEMMA 4. If $\sum_{p=0}^{\infty} a_{p} z^{p}$ converges for $|z|<1$ and $\left|\sum_{p=0}^{\infty} a_{p} z^{p}\right| \leqq 1$, for $|z|<1$, then $\left|a_{x}\right| \leqq 1$ for $p=0,1, \cdots$.

Proof. Let $0<r<1$ and $\epsilon>0$. There exists $N>0$ such that if $n>N,\left|\sum_{p=0}^{n} a_{p} r^{p} z^{p}\right|<1+\epsilon$ for $|z| \leqq 1$. By Lemma $3,\left|(1+\epsilon)^{-1} a_{p} r^{p}\right|$ $\leqq 1$ for $p=0,1, \cdots, n$. Thus $\left|a_{p}\right| \leqq 1$ for all $p$.

Lemma $5{ }^{1}$ Let $f(z)$ be differentiable and $|f(z)|<1$ for $|z|<1$. Let $f_{0}(z)=f(z)$ and $f_{p+1}(z)=\left[e_{p}-f_{p}(z)\right]\left(z\left[1-\bar{e}_{p} f_{p}(z)\right]\right)^{-1}$ where $e_{p}=f_{p}(0)$. Then either

(1) $\left|e_{p}\right|<1$ for all $p$ and $f_{p}$ will be defined and differentiable for $|z|$ $<1$ with $\left|f_{p}(z)\right|<1$ for $|z|<1$ and $p=0,1, \cdots$ or

(2) $\left|e_{p}\right|<1$ for $p=0,1, \cdots(k-1)$ with $\left|e_{k}\right|=1$ and $f_{p}$ will be defined and differentiable for $|z|<1$ and $p=0,1, \cdots, k$ with $\left|f_{p}(z)\right|$ $<1$ for $|z|<1$ and $p \leqq k-1$ and $f_{k}(z)=$ constant of modulus one.

Proof. If $h$ is a differentiable function with $|h(z)|<1$ for $|z|<1$ and $e=h(0)$, then $\left|[e-h(z)][1-\bar{e} h(z)]^{-1}\right|<1$ for $|z|<1$ as is well known. Now, if $g(z)=[e-h(z)](z[1-\bar{e} h(z)])^{-1}$, with $g(0)$ defined to be $-h^{\prime}(0)\left(1-|e|^{2}\right)^{-1}$, then $g$ is continuous at $z=0$ and by Lemma 2 is also differentiable there. Note that $|g(z)| \leqq|z|^{-1}$ for $0<|z|<1$ and thus by Lemma $1,|g(z)| \leqq 1$ for $|z|<1$. If $|g(z)|<1$ for $|z|<1$, then $h$ may be replaced by $g$ and the process continued. If there exists $z_{0}$ with $\left|z_{0}\right|<1$ and $\left|g\left(z_{0}\right)\right|=1$, then by Lemma $1, g$ is a constant of modulus one. This establishes Lemma 5.

Lemma 6. Suppose that, under the hypothesis of Lemma 5, the first case holds and let, for $|z|<1$ and $\left|t_{p}\right| \leqq 1$ where $t_{p}$ is arbitrarily chosen for $p=0,1, \cdots, s_{p}\left(z, t_{p}\right)=\left[e_{p}-z t_{p}\right]\left[1-\bar{e}_{p} z t_{p}\right]^{-1}=e_{p}$ $+\left(1-\left|e_{p}\right|^{2}\right) z\left[\bar{e}_{p} z-t_{p}^{-1}\right]^{-1}$ and $S_{p}\left(z, t_{p}\right)=S_{p-1}\left(z, s_{p}\left(z, t_{p}\right)\right), p=1,2, \cdots$, and $S_{0}\left(z, t_{0}\right)=s_{0}\left(z, t_{0}\right)$. Then $\left|S_{p}\left(z, t_{p}\right)\right|<1$ and $S_{p}\left(z, f_{p+1}\right)=f_{0}(z)$ for $p=0,1, \cdots$.

${ }^{1}$ Schur's technique is outlined on pages $285-288$ of [3], where some basic identities from continued fraction theory and Schwarz's lemma are used. Hence Lemma 5 and Lemma 6 of this paper do no more than set up. the continued fraction expansion for $f(z)$, and the proof of Lemma 7 avoids the use of the fundamental determinant formula for continued fractions. 
Proof. Since $\left|e_{p}\right|<1$ and $\left|s_{p}\left(z, t_{p}\right)\right|<1$, the first conclusion is immediate. For the second conclusion, note that $s_{p}\left(z, f_{p+1}(z)\right)=f_{p}(z)$ for $p=0,1, \cdots$ Thus

$$
\begin{aligned}
S_{p}\left(z, f_{p+1}(z)\right) & =S_{p-1}\left(z, s_{p}\left[z, f_{p+1}(z)\right]\right)=S_{p-1}\left(z, f_{p}(z)\right) \cdots \\
& =S_{0}\left(z, f_{1}(z)\right)=s_{0}\left(z, f_{1}(z)\right)=f_{0}(z) .
\end{aligned}
$$

LemMa 7. Under the hypotheses of Lemmas 5 and 6 we have, for $p=0,1, \cdots$, that $S_{p}\left(z, t_{p}\right)=\sum_{m=0}^{\infty} a_{m}\left(p, t_{p}\right) z^{p}$ with the series converging to $S_{p}\left(z, t_{p}\right)$ for $|z|<1$ and, moreover, the coefficients $a_{m}\left(p, t_{p}\right)$ are independent of $p$ and $t_{p}$ when $p \geqq m$ and, finally, $\left|a_{m}\left(p, t_{p}\right)\right| \leqq 1$ for all $p$ and $m$.

Proof. The functions $S_{p}\left(z, t_{p}\right)$ are rational functions of $z$ with a nonzero denominator provided $|z|<1$ and, consequently have a power series expansion for $|z|<1$. Now $a_{0}\left(p, t_{p}\right)=S_{p}\left(0, t_{p}\right)=f(0)$. Hence, suppose first that the coefficients are independent of $t_{p}$ for $m \leqq p$ and $p \leqq n$. (Suppose, in fact, that this is true regardless of what function $f$ is used originally.) By definition, $S_{n+1}\left(z, t_{n+1}\right)$ $=S_{n}\left(z, s_{n+1}\left(z, t_{n+1}\right)\right)=\cdots=s_{0}\left(z, s_{1}\left(z, s_{2}\left(z, \cdots, s_{n+1}\left(z, t_{n+1}\right)\right)\right) \cdots\right)$ and this is $e_{0}+\left(1-|e|^{2}\right) z\left[e_{0} z-K^{-1}\left(z, t_{n+1}\right)\right]^{-1}$ where $K\left(z, t_{n+1}\right)$ $=s_{1}\left(z, s_{2}\left(z, s_{3}\left(z, \cdots, s_{n+1}\left(z, t_{n+1}\right)\right)\right) \cdots\right)$. By the inductive hypothesis (with $f$ replaced by $f_{1}$ ) the first $n$ terms of the power series of $K\left(z, t_{n+1}\right)$ are independent of $t_{n+1}$. Thus the first $n+1$ terms of the power series expansion of $e_{0}+\left(1-\left|e_{0}\right|^{2}\right) z\left[\bar{e}_{0} z-K^{-1}\left(z, t_{n+1}\right)\right]$ are independent of $t_{n+1}$.

Now to show that $a_{m}\left(p, t_{p}\right)$ is independent of $p$ for $p \geqq m$, simply note that, since $S_{p+1}\left(z, t_{p+1}\right)=S_{x}\left(z, s_{p+1}\left(z, t_{p+1}\right)\right), a_{m}\left(p+1, t_{p+1}\right)$ $=a_{m}\left(p, s_{p+1}\left(z, t_{p+1}\right)\right)$ for all $m$ and $p$. But for $p \geqq m$, these are independent of $t_{p+1}$, so that $a_{m}(p+1)=a_{m}(p)$ for $p \geqq m$. Finally, $\left|S_{p}\left(z, t_{p}\right)\right|$ $<1$ by Lemma 6 so that $\left|a_{m}\left(p, t_{p}\right)\right| \leqq 1$ by Lemma 4 .

This completes the proof of Lemma 7.

Let us now define, in view of Lemma 7,

$$
a_{m}=a_{m}\left(p, t_{p}\right)
$$

for $m=0,1, \cdots, p$ and $p=0,1, \cdots$. then

ThEOREM. If $f$ is differentiable for $|z|<1$ and $|f(z)|<1$ for $|z|<1$,

$$
f(z)=\sum_{m=0}^{\infty} a_{m} z^{m}
$$

for $|z|<1$ and $\left|a_{m}\right| \leqq 1$ for all $m$. 
Proof. Let us suppose that case 1 of Lemma 5 holds. The coefficients $a_{m}$ are defined as above so that by Lemma $7,\left|a_{m}\right| \leqq 1$. Now when $p<q$,

$$
\begin{aligned}
\left|S_{q}\left(z, t_{q}\right)-S_{p}\left(z, t_{p}\right)\right| & =\left|\sum_{m=p+1}^{\infty} a_{m}\left(q, t_{q}\right) z^{m}-\sum_{m=p+1}^{\infty} a_{m}\left(p, t_{p}\right) z^{m}\right| \\
& \leqq 2 r^{p+1}(1-r)^{-1}
\end{aligned}
$$

whenever $|z| \leqq r<1$. Thus $\lim _{p} S_{p}\left(z, t_{p}\right)$ exists uniformly on compact subsets of $|z|<1$ and is independent of the choice of $t_{p}$. By Lemma 6, $S_{\mathfrak{y}}\left(z, f_{p+1}(z)\right)=f_{0}(z)=f(z)$, thus $\lim _{p} S_{p}\left(z, t_{p}\right)=f(z)$. Now since

$$
\begin{aligned}
\left|\sum_{m=0}^{\infty} a_{m} z^{m}-\sum_{m=0}^{\infty} a_{m}\left(p, t_{p}\right) z^{m}\right| & =\left|\sum_{m=p+1}^{\infty} a_{m} z^{m}-\sum_{m=p+1}^{\infty} a_{m}\left(p, t_{p}\right) z^{m}\right| \\
& \leqq 2 r^{p+1}(1-r)^{-1}
\end{aligned}
$$

it follows that $f(z)=\sum_{m=0}^{\infty} a_{m} z^{m}$.

Let us now suppose that case 2 of Lemma 5 holds so that $f_{p}(z)$ is defined only for $p=0,1, \cdots, k$ with $f_{k}(z)=e^{i \alpha}$. Also, $s_{p}$ and $S_{p}$ will be defined only for $p=0,1, \cdots, k$. Simply notice, as before, that $f(z)=S_{k-1}\left(z, f_{k}(z)\right)=S_{k-1}\left(z, e^{i \alpha}\right)=\sum_{m=0}^{\infty} a_{m}\left(k-1, e^{i \alpha}\right) z^{m}$. Thus, in this case, the coefficients are constants and $a_{m}$ is defined to be $a_{m}\left(k-1, e^{i \alpha}\right)$ and $f(z)=\sum_{m=0}^{\infty} a_{m} z^{m}$ as before.

CoRollaRY. If $f$ is differentiable in a region $R$, then $f^{\prime}$ is continuous and differentiable in $R$.

\section{BIBLIOGRAPHY}

1. P. Porcelli and E. H. Connell, $A$ proof of the power series expansion without Cauchy's formula, Bull. Amer. Math. Soc. 67 (1961), 177-181.

2. J. Schur, Über Potenzreihen, die im Innern des Einheitskreises beschrankt sind, Crelle's Journal 147 (1917), 205-232.

3. H. S. Wall, Analytic theory of continued fractions, Van Nostrand, New York, 1948.

4. G. T. Whyburn, Topological analysis, Princeton Univ. Press, Princeton, N. J., 1958.

Louisiana State University 